\title{
Severe Hypercalcemia as the Presenting Sign of Tuberculosis
}

\author{
Michael J. Kavanaugh ${ }^{\mathrm{a}, \mathrm{b}}$, Mary F. Bavaro ${ }^{\mathrm{a}}$, Robert V. Barthel ${ }^{\mathrm{a}}$, Ryan C. Maves ${ }^{\mathrm{a}}$, \\ Harold L. Groff ${ }^{a}$
}

\begin{abstract}
Severe hypercalcemia due to tuberculosis (TB) is rare. We describe a case of a 25-year-old male who was found to have a serum calcium level $15.4 \mathrm{mg} / \mathrm{dL}$ at a routine appointment prompting admission. Chest imaging demonstrated hilar lymphadenopathy and airspace opacities principally involving the lingula. Multiple sputum smears were negative for acid-fast bacilli (AFB), but cultures of sputum subsequently grew Mycobacterium tuberculosis. Despite appropriate therapy for $\mathrm{TB}$, his serum calcium failed to normalize, prompting initiation of prednisone with rapid improvement. In granulomatous diseases, including TB, inflammation is mediated in part by elevated calcitriol released from macrophages, with resulting rises in serum calcium concentrations. Glucocorticoids are commonly used as adjunctive therapy for TB meningitis and pericarditis and this case emphasizes the potential role for steroids in refractory hypercalcemia.
\end{abstract}

Keywords: Tuberculosis; Hypercalcemia; Glucocorticoids

\section{Introduction}

Hypercalcemia in granulomatous diseases is well described but rarely symptomatic [1], with elevated serum calcium levels reported in up to $16 \%$ of patients with tuberculosis (TB) in the United States [2]. Hypercalcemia in TB may be more common in tropical settings, however, with up to $27.5 \%$ of TB patients in Nigeria presenting with hypercalcemia in a recent series. Of the hypercalcemic subset, only $12 \%$ experienced symptoms [1]. No difference in hypercalcemia incidence has been described between pulmonary and extrapulmonary TB [1], although the highest reported serum calcium level in the literature to date was $15 \mathrm{mg} / \mathrm{dL}$ in a patient with tuberculous

Manuscript accepted for publication July 01, 2015

aDivision of Infectious Diseases, Department of Internal Medicine, Naval Medical Center San Diego, San Diego, CA, USA

bCorresponding Author: Michael J. Kavanaugh, Naval Medical Center San Diego, 34800 Bob Wilson Dr, San Diego, CA 92134, USA.

Email: mkav4321@yahoo.com

doi: http://dx.doi.org/10.14740/jmc2220w peritonitis [3].

Hypercalcemia in granulomatous diseases is proposed to be the result of extra-renal conversion of dietary 25-hydroxyvitamin D3 to the active 1,25-vitamin D3 (calcitriol), independent of parathyroid hormone (PTH), a mechanism best described in sarcoidosis [4]. Calcitriol has a favorable adaptive response by inhibiting IL-2 and interferon- $\gamma$ and mediating inflammation [5]. Elaboration of parathyroid hormone-related protein (PTHrP) by granulomata may represent an additional mechanism for hypercalcemia; this mechanism has been described in sarcoidosis [6] and coccidiomycosis [7] but not in TB to date. Hypercalcemia, as a manifestation of sarcoidosis, frequently resolves with glucocorticoids, but to our knowledge, this is only the third case report of steroid treatment for hypercalcemia secondary to TB.

\section{Case Report}

A 25-year-old Filipino male with a history of developmental delay and chronic renal disease with proteinuria presented to his nephrologist for a routine appointment. The patient had no subjective complaints, but record review confirmed a weight loss of $10 \mathrm{~kg}$ compared to the prior year. He had a dry cough but denied hemoptysis or shortness of breath. On examination, he was under-nourished with a body mass index of 18.4 in addition to bilateral cervical lymphadenopathy. A serum chemistry panel obtained on the day of evaluation revealed hypercalcemia $(15.4 \mathrm{mg} / \mathrm{dL}$, normal $8.9-10.4 \mathrm{mg} / \mathrm{dL})$.

The patient was admitted to the intensive care unit for intravenous hydration and diuretic therapy. A chest radiograph demonstrated hilar lymphadenopathy with a lingular airspace opacity; a contrast-enhanced computed tomogram (CT) of the chest and neck demonstrated additional tree-and-bud opacities (Fig. 1) with diffuse cervical and mediastinal lymphadenopathy (Fig. 2). Diagnostic studies were significant for a positive interferon-gamma release assay for tuberculous antigen, multiple negative sputum smears for acid-fast bacilli (AFB), and a negative serum human immunodeficiency virus enzyme immunoassay. His PTH was appropriately low at $2.91 \mathrm{pg} / \mathrm{mL}$ (normal $15-65 \mathrm{pg} / \mathrm{mL}$ ) with a normal PTHrP of $20 \mathrm{pg} / \mathrm{mL}$ (normal $14-27 \mathrm{pg} / \mathrm{mL}$ ). However, he was noted to have an elevated level of 1,25-hydroxy-vitamin D3 (84 nmol/L, normal $18-72 \mathrm{nmol} / \mathrm{L}$ ) and a low level of 25-hydroxy-vitamin D3 


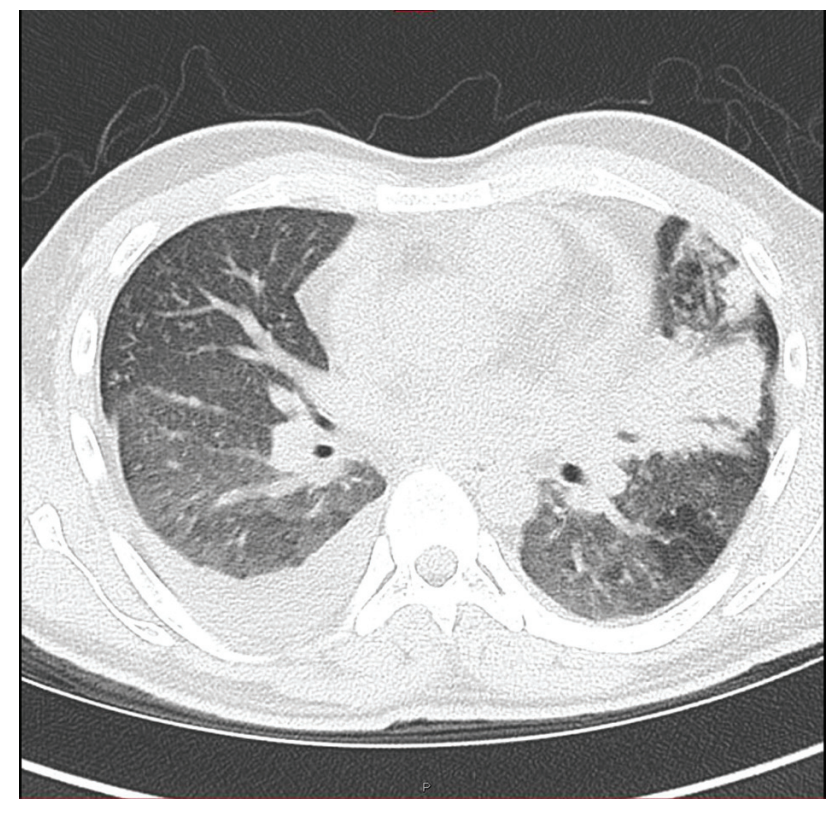

Figure 1. Contrast-enhanced computed tomogram (CT) of the chest displaying enlarged lymph nodes, tree-in-bud pulmonary opacities and dense consolidation within the lingula.

(12 nmol/L, normal 35 - $80 \mathrm{nmol} / \mathrm{L}$ ). A lymph node biopsy revealed granulomatous inflammation, without evidence of AFB by Ziehl-Niessen staining.

Based on high clinical suspicion for active TB, the patient was empirically started on treatment for TB with rifampin, isoniazide (INH), pyrazinamide and ethambutol during the hospital admission. Seven weeks after presentation, cultures of sputum and the lymph node grew Mycobacterium tuberculosis with isolated resistance to INH. Moxifloxacin was subsequently added to his regimen and INH discontinued. His serum calcium levels normalized at discharge to $8.1 \mathrm{mg} / \mathrm{dL}$ following rehydration with furosemide diuresis. He remained asymptomatic and was afebrile without cough and achieved a $3-\mathrm{kg}$ weight gain over the first 2 months of treatment. Surveillance sputum AFB cultures after 8 weeks of therapy revealed no growth.

Despite his apparent improvement, his cervical lymphadenopathy persisted, and his calcium levels increased to $10.3 \mathrm{mg} /$ $\mathrm{dL}$ despite use of oral furosemide. After 6 months of therapy, he was treated with prednisone $20 \mathrm{mg} / \mathrm{day} \times 21$ days followed by a 7-day taper which resolved his calcium issue to $8.8 \mathrm{mg} /$ $\mathrm{dL}$ and his follow on calcitriol had reduced to $16 \mathrm{nmol} / \mathrm{L}$. His calcium levels remained within normal limits over the next several months of treatment, he regained $15 \mathrm{~kg}$ and his lymph nodes ultimately regressed.

\section{Discussion}

Our patient had chronic and asymptomatic hypercalcemia with adenopathy that persisted despite antituberculous therapy and loop diuretics, with an excellent clinical response to prednisone. Data regarding the use of glucocorticoids as a therapy for TB-related hypercalcemia are limited. Previous case re-

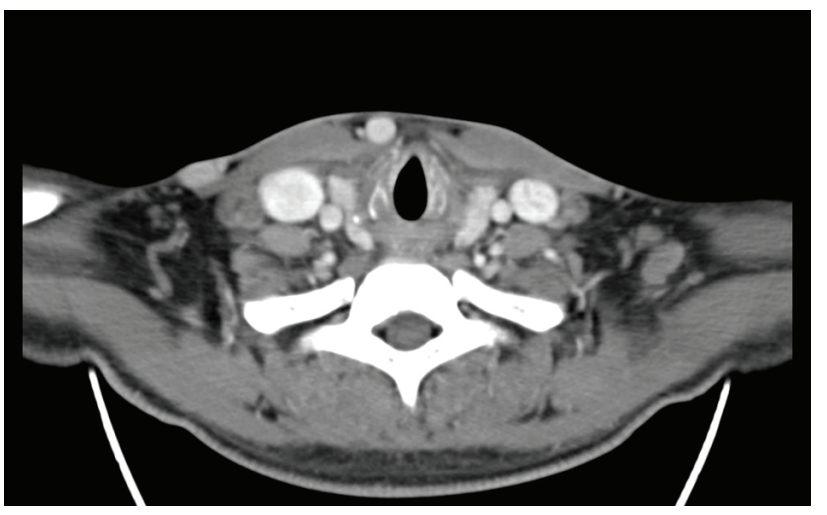

Figure 2. Contrast-enhanced CT of the neck displaying "diffuse cervical and mediastinal lymphadenopathy of inhomogeneous necrotizing appearing lymphadenopathy".

ports have described pulmonary TB with symptomatic, refractory hypercalcemia $[8]$ and skeletal TB with hypercalcemia [9] successfully treated with glucocorticoids. Although not routine practice outside of specific syndromes such as meningitis or pericarditis, there are increasing data regarding the use of glucocorticoids as adjunctive therapy in TB. A recent meta-analysis reported an overall reduction in TB mortality of $17 \%$ (RR: 0.83 ) with the use of adjunctive glucocorticoids, including a statistically significant mortality benefit with meningitis and pericarditis. These conclusions are limited by the inclusion of both randomized and non-randomized trials and a lack of overall statistical significance [10]. Additionally, the randomized trials utilized older regimens which are not consistent with today's standard of care and no study specifically identified either TB adenopathy or hypercalcemia. This case displays the potential utility of glucocorticoids as a treatment option in refractory TB adenopathy with hypercalcemia. Aside from meningitis and pericarditis, further data are needed to better evaluate the role of glucocorticoids in the treatment of the complications of TB including hypercalcemia.

\section{Disclaimer}

The authors are employees of the United States Government. This work was prepared as part of their official duties. Title 17 U.S.C. $\$ 105$ provides that "Copyright protection under this title is not available for any work of the United States Government". Title 17 U.S.C. $\S 101$ defines a US Government work as a work prepared by a military service member or employee of the US Government as part of that person's official duties. The views expressed in this article are those of the authors and do not necessarily reflect the official policy or position of the Departments of the Navy, the Department of Defense, nor the US Government.

\section{References}

1. Dosumu EA, Momoh JA. Hypercalcemia in patients with newly diagnosed tuberculosis in Abuja, Nigeria. Can 
Respir J. 2006;13(2):83-87.

2. Abbasi AA, Chemplavil JK, Farah S, Muller BF, Arnstein AR. Hypercalcemia in active pulmonary tuberculosis. Ann Intern Med. 1979;90(3):324-328.

3. Soofi A, Malik A, Khan J, Muzaffar S. Severe hypercalcemia in tuberculosis. J Pak Med Assoc. 2004;54(4):213215.

4. Inui N, Murayama A, Sasaki S, Suda T, Chida K, Kato S, Nakamura H. Correlation between 25-hydroxyvitamin D3 1 alpha-hydroxylase gene expression in alveolar macrophages and the activity of sarcoidosis. Am J Med. 2001;110(9):687-693.

5. Conron M, Young C, Beynon HL. Calcium metabolism in sarcoidosis and its clinical implications. Rheumatology (Oxford). 2000;39(7):707-713.

6. Zeimer HJ, Greenaway TM, Slavin J, Hards DK, Zhou H, Doery JC, Hunter AN, et al. Parathyroid-hormone-related protein in sarcoidosis. Am J Pathol. 1998;152(1):17-21.

7. Fierer J, Burton DW, Haghighi P, Deftos LJ. Hypercalcemia in disseminated coccidioidomycosis: expression of parathyroid hormone-related peptide is characteristic of granulomatous inflammation. Clin Infect Dis. 2012;55(7):e61-66.

8. Rizwan A, Islam N. Middle aged male with pulmonary tuberculosis and refractory hypercalcemia at a tertiary care centre in South East Asia: a case report. Cases J. 2009;2:6316.

9. Braman SS, Goldman AL, Schwarz MI. Steroid-responsive hypercalcemia in disseminated bone tuberculosis. Arch Intern Med. 1973;132(2):269-271.

10. Critchley JA, Young F, Orton L, Garner P. Corticosteroids for prevention of mortality in people with tuberculosis: a systematic review and meta-analysis. Lancet Infect Dis. 2013;13(3):223-237. 